\title{
Abandon all models
}

\section{Leslie Sage}

IN April 1920, a great astronomical debate was held between Harlow Shapley and Heber D. Curtis in the auditorium of the Museum of Natural History in Washington DC. That debate centred on the nature of the 'spiral nebulae', which we now know are other galaxies, but which many astronomers at that time (including Shapley) held to be similar to the gaseous nebulae surrounding massive young stars and now identified as regions of ionized hydrogen.

Partly to mark the 75 th anniversary of the event, a debate on one of today's most contentious astronomical problems was held in the same auditorium on 22 April this year. Twenty-six years after the discovery of bursts of gamma-ray emission from space, the source of these very-highenergy photons is as mysterious as ever. Donald Lamb and Bohdan Paczyński, leading proponents of the two major prevailing views, put forward their opinions on the relevant issues.

Paczyński started the debate by stating that there are two camps, but that they are not the ones usually recognized. He said that there are those who acknowledge that they have no idea what is causing the bursts, and those who do not acknowledge that basic fact. All we really know about the gamma-ray bursters (GRBs) is that they are distributed more or less isotropically across the sky. There are only about 1,100 recorded bursts, making it difficult to determine whether the distribution is truly isotropic, and therein lies the source of the controversy.

Most objects associated with our Galaxy are not distributed isotropically, except for a restricted sample of nearby stars. There is an inherent anisotropy built into the Milky Way because of its disk-like structure, which is easily seen in the bright band of stars across the night sky. The only other objects in the Universe that are distributed in a relatively uniform fashion across the sky are very distant galaxies. (Although there seem to be structures in the distribution of galaxies, we will regard their distribution as isotropic for simplicity.) Even the globular clusters, which are distant compact groups of a million or more stars orbiting the centre of the Milky Way, are not isotropically distributed from our perspective in the outer part of the Milky Way. They are definitely clustered around the centre, in the direction of the constellation of Sagittarius.

This relative isotropy suggested to many people that the GRBs are either pretty local (originating within a few hundred parsecs of the Sun) or lie at distances comparable to the most distant galaxies. As instruments have improved, particu- larly with the launching of the Compton Gamma-Ray Observatory by NASA in 1991, the so-called cosmological origin (perhaps associated with very distant galaxies) has come to be favoured, because the distribution has remained isotropic. But on the opposite side of the debate, the 'local' option, championed by Don Lamb, now favours sources distributed in an extended halo around the Milky Way, with an average distance of $100 \mathrm{kpc}$.

As Lamb pointed out, the current data are consistent with this interpretation. Paczyński countered that the critical test will arise when we have more data, which should allow us to see an anisotropy in the distribution arising from our position 8 $\mathrm{kpc}$ from the centre of the Milky Way. In particular, there should be an excess of sources in the direction of M31, the nearest giant spiral galaxy to us. M31 lies at a distance of only $600 \mathrm{kpc}$, so if we can detect GRBs with an average distance of $100 \mathrm{kpc}$ around the Milky Way, we should see an excess of relatively faint sources in the direction of M31. Lamb admitted that if this excess is not seen, and the distribution of sources continues to be isotropic around us, then he will have to abandon his position.

Paczyński suggested that the best way to make progress towards understanding the GRBs is to abandon all models. The astronomical literature is rife with proposed explanations, which range from colliding comets to merging neutron stars. More speculative sources include the exhaust from warp-drive engines. Paczyński maintained, however, that preconceived notions of what might be causing the bursts are biasing the debate about their distance scale. He recommended that we discard all current models until we have more information.

Despite the entertaining points made by both Lamb and Paczyński during the debate, I doubt that anyone entering the auditorium with preconceived notions left with a different opinion. The data are only preliminary, and some will undoubtedly prove to be wrong or misleading.

Shapley, who is generally considered to have 'won' the debate seventy-five years ago, was proven wrong within five years by Edwin Hubble. The decision about who won this year's debate seems less clear, although the majority opinion is in favour of a cosmological origin of the GRBs. Will history repeat itself, quickly demonstrating that the GRBs arise from a local population of sources? One thing is certain - the Universe yet holds many surprises for us.

Leslie Sage is an assistant editor of Nature.
DAEDALUS

\section{Watch this space}

THE human eye trembles all the time, scanning the focused image over its retina at $30-80 \mathrm{~Hz}$. This scan is crucial to vision. If an optical target is vibrated in synchronism with the scan, its image can be stabilized on the retina. It promptly disappears from view.

In principle, therefore, an agile dancer might vanish from a watcher's gaze, simply by shimmying at his optical scanning frequency. Daedalus is now making this scheme more practical. His 'cloak of invisibility' is a bulky, allenveloping garment made of a liquidcrystal display material. An invisible infrared beam is projected through the fabric; it bounces off the watcher's eyes and retrieves their scanning motion. This is used to vibrate, not the cloak or its wearer, but the pattern displayed on the cloak. As synchronization locks in, it vanishes from the watcher's gaze.

What will the watcher make of this sudden blank in his field of view? He will not even be aware of it. The visual system automatically fills in a retinally stabilized gap with some plausible interpolation of the surroundings, just as it fills in our blind spot. This is big enough to take the images of 150 full moons, but we never notice it. Similarly, anyone looking at the cloak of invisibility will unknowingly extend the background scene right across it, and see nothing at all.

The cloak of invisibility will appeal to policemen, private detectives, spies, criminals, extramarital adventurers and many others wishing to blush unseen. Its main snag is that it can work perfectly against one watcher only. Two, each with his own scanning frequency, cannot be simultaneously unsighted. Even so, they may both be fooled. The watcher 'in sync' will see nothing at all. The other will see the wearer vanishing and reappearing at the beat frequency between the cloak's vibration and that of his own eyes. This could be just as baffling.

Indeed, Daedalus is planning a simpler cloak of invisibility whose pattern just vibrates unsynchronized at a range of frequencies around $50 \mathrm{~Hz}$, varying from one point to another. Any watcher will then see the wearer broken up by wandering regions of invisibility, each filled in with some mental construct of his own. The static disruption of an expected outline is baffling enough: the dazzle-patterns worn by the ships of World War I made them hard to see or identify even against a blank horizon. Disruptions drifting with time will be far worse, and will utterly confuse the witnesses. Even if they manage to interpret the apparition correctly, their claims will seem incredible in court.

David Jones 